\title{
A Multi-functional Micro-machined Gyroscope for Rotating Aircraft
}

\author{
Qingwen Yan ${ }^{1,2, a}$, Fuxue Zhang ${ }^{1, b}$ and Wei Zhang ${ }^{1, c}$ \\ ${ }^{1}$ Sensing Technique Research Center, Beijing Information Science and \\ Technology University, Beijing, China \\ ${ }^{2}$ School of Electronic Engineering, Beijing University of Posts and \\ Telecommunications, Beijing, China

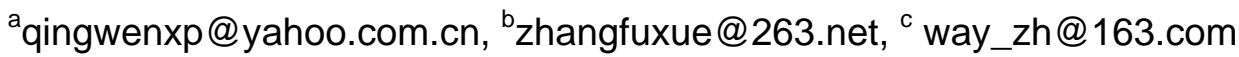

\begin{abstract}
Keywords: silicon micromachining; gyroscope; rotating aircraft
\end{abstract}
\begin{abstract}
In this paper, we present recent work on the design, fabrication, and packaging of a new gyroscope, by silicon micromachining, for the autopilot of rotating aircraft. The multi-functional micro-machined gyroscope generates the gyroscope effect driven by the roll of rotating carrier, and sense the roll frequency in the longitudinal axis direction of rotating carrier and the transverse angular rate in the perpendicular direction of longitudinal axis of rotating carrier. In addition, the transverse angular rate can be decomposed into the yaw angular rate and pitch angular rate with the geographic coordinates of reference. Therefore, multi-functional micro-machined gyroscope can sense angular rate in three directions, and the signal of gyroscope can be calculated into the five parameters, including the transverse angular rate, azimuth angle, yaw angular rate, pitch angular rate and roll frequency. The new gyroscope has the functions of three the general rate gyroscopes mounted vertically and fits for the attitude control of rotating aircraft.
\end{abstract}

\section{Introduction}

Improvement of control and stability system of aircraft largely depends on the improvement of accuracy and reliability of instrument for measuring the space movement of aircraft. Meanwhile the airborne equipment are seriously crowded in the cabin, many attentions are paid on the miniaturization of mechanical and electrical instruments which include gyroscope and stability systems. Therefore a new gyroscope should be small size, light weight, low power consumption and low prices. In 1990s, a wide variety of silicon micro-machined gyroscope appeared with the development of microelectronics technology. But most of the silicon micro-machined gyroscopes use static electrical force to drive the mass and sense the angular rate of the carrier in the sense mode [1, 2]. In general the performance of vibrating rate gyroscope is strongly influenced by two characteristic modes of motion: (i) the first or fundamental mode defining the drive frequency, and (ii) the secondary mode defining the sense motion [3,4]. Compared with those gyroscopes, a new gyroscope driven by the rotating carrier is presented, which only has sense motion mode $[5,6]$. The drive force comes from the roll of the carrier, so the signal of gyroscope contains the information of the roll frequency of the carrier.

\section{Design and Fabrication}

The silicon pendulum of the micro-machined gyroscope contains the pendulum with many damping slots suspended by two torsion girders that are anchored to the outer frame, shown in Fig.1. By applying the roll frequency, $\dot{\varphi}$, to the outer frame, the silicon pendulum rotates with the outer frame about the $\mathrm{Z}$ axis. An angular rate, $\Omega$, applied about the $\mathrm{X}$ axis, generates a Coriolis force that acts to push the pendulum in and out of the frame of oscillation, i.e. sense motion. 
In general the sense motion is a forced damped vibration and can be described for a rotational angle $\alpha$, torque $K_{T}$, the moment of inertia $J_{X}, J_{Y}, J_{Z}$ and damping factor $\zeta$. The roll frequency and transverse angular rate of the carrier is $\dot{\varphi}$ and $\Omega$.

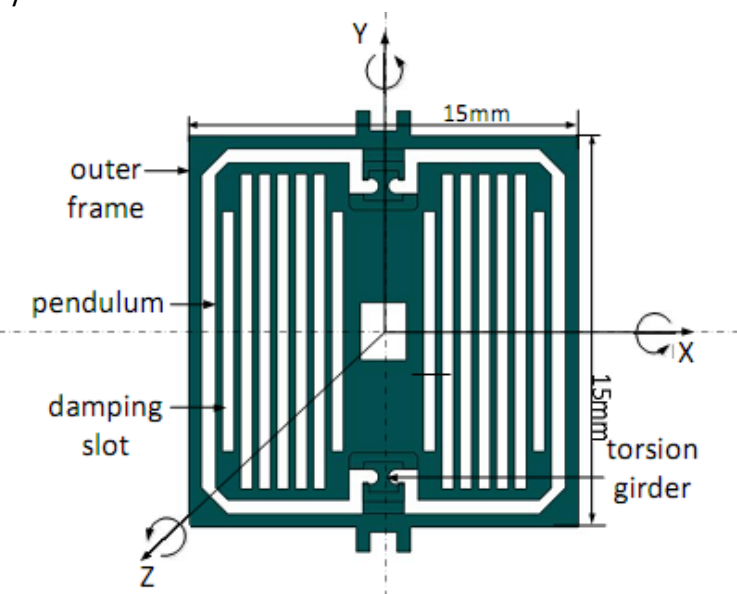

Fig. 1 Structure of silicon pendulum of micro-machined gyroscope

$$
J_{Y} \ddot{\alpha}+\zeta \dot{\alpha}+\left[\left(J_{Z}-J_{X}\right) \dot{\varphi}^{2}+K_{T}\right] \alpha=\left(J_{Z}+J_{Y}-J_{X}\right) \Omega \dot{\varphi} \cos (\dot{\varphi} t)
$$

The stationary solution of Eq. 1 is [7]

$$
\alpha=\frac{\left(J_{Z}+J_{Y}-J_{X}\right) \dot{\varphi}}{\sqrt{\left[\left(J_{Z}-J_{Y}-J_{X}\right) \dot{\varphi}^{2}+K_{T}\right]^{2}+(\zeta \dot{\varphi})^{2}}} \Omega \cos (\dot{\varphi} t-\beta)
$$

where $\beta$ is the phrase difference between the oscillation phrase and the direction of the transverse angular rate.

The rotational angle of silicon pendulum is determined by the Coriolis force, and the oscillation frequency of silicon pendulum is equal to the roll frequency of the carrier.

Starting from a standard 4" two-side polished silicon wafer, a thick thermal oxide layers are grown on both sides of the wafer. In the first lithography, silicon oxide etching and silicon etching step, the pendulum thickness and the outer frame are realized. Another thick thermal oxide is grown. Onto this pendulum, the damping slots are opened in the second lithography, silicon oxide etching and silicon etching step. The pendulum is released in the third and fourth lithography, silicon oxide etching and silicon etching step. The third thick thermal oxide is grown. The torsion girders are realized in fifth lithography, silicon oxide etching and silicon etching step. Two electrode plates are glued on the silicon chip encapsulating the whole pendulum element. The shell and lid provide a hermetical sealing, shown in Fig.2.

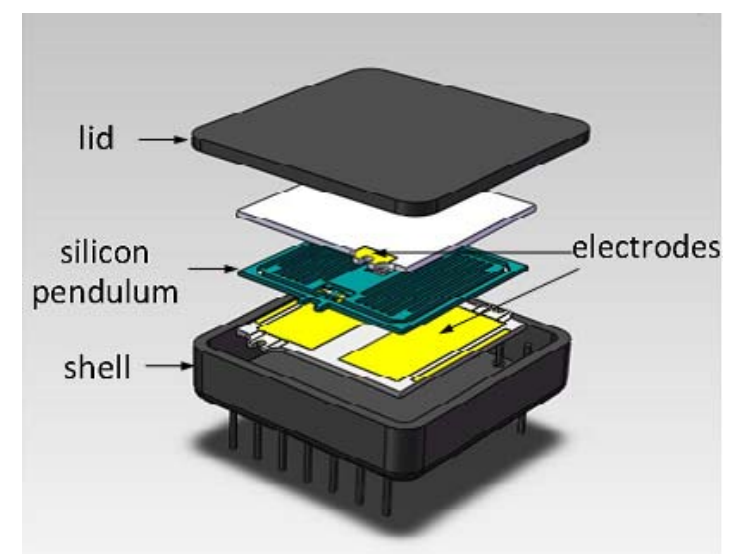

Fig.2 Structure of micro-machined gyroscope 
The gyroscope signal is the amplitude modulation ware. The change of signal amplitude reflects the change of input angular rate, and the change of signal frequency reflects the change of the roll frequency of the rotating aircraft.

The signal of micro-machined gyroscope is

$$
U=\frac{K_{T S}\left(J_{Z}+J_{Y}-J_{X}\right) \dot{\varphi}}{\sqrt{\left[\left(J_{Z}-J_{Y}-J_{X}\right) \dot{\varphi}^{2}+K_{T}\right]^{2}+(\delta \dot{\varphi})^{2}}} \Omega \cos (\dot{\varphi} t-\beta)
$$

where $K_{T S}$ is the capacitance pick-up factor. Eq. 3 can be simplified into

$$
U=K(\dot{\varphi}) \Omega \cos (\dot{\varphi} t-\beta)
$$

where $K(\dot{\varphi})$ is the scale factor of micro-machined gyroscope, which is measured with different roll frequency and different transverse angular rate. Therefore $K(\dot{\varphi})$ is the polynomial of the roll frequency.

$$
K(\dot{\varphi})=a_{0}+a_{1} \dot{\varphi}+a_{2} \dot{\varphi}^{2}+a_{2} \dot{\varphi}^{3} \cdots
$$

The Hilbert transform of Eq. (4) is

$\hat{U}(t)=K(\dot{\varphi}) \Omega \sin (\dot{\varphi} t-\beta)$

where $\hat{U}$ is Hilbert transform of $U$.

The roll angle of the carrier is obtained by divide Eq. (6) by Eq. (4). The roll frequency of the carrier is the differential of the roll angle.

$$
\dot{\varphi} t-\beta=\arctan \left(\frac{\hat{U}}{U}\right)
$$

The transverse angular rate $\Omega$ is

$$
\begin{aligned}
& U^{2}(t)+\hat{U}^{2}(t)=K^{2}(\dot{\varphi}) \Omega^{2} \cos ^{2}(\dot{\varphi} t-\beta)+K^{2}(\dot{\varphi}) \Omega^{2} \sin ^{2}(\dot{\varphi} t-\beta) \\
& \Omega=\frac{1}{K(\dot{\varphi})} \sqrt{U^{2}(t)+\hat{U}^{2}(t)}
\end{aligned}
$$

The transverse angular rate can be decomposed into the yaw angular rate and pitch angular rate with the geographic coordinates of reference. A micro-machined accelerator is an ideal geographic coordinates of reference, because it can sense the direction of gravity and not be influenced by the angular rate. The signal of accelerator is

$$
U_{a}(t)=A \sin (\dot{\varphi} t-\chi)
$$

where $A$ is a constant amplitude of signal which is only related with the gravity; $\chi$ is the phrase of signal which induces the direction of the gravity and is used as the geographic coordinates of reference.

The azimuth angle of transverse angular rate is

$\theta=\beta-\chi$

The yaw angular rate is

$\Omega_{Y}=\Omega \sin (\theta)$

The pitch angular rate is

$\Omega_{P}=\Omega \cos (\theta)$

\section{Experiments and Results}

Normally the movement of rotating aircraft is the coning motion, which the rotating aircraft rolls about its longitudinal axis and its head moves in eclipse, shown in Fig. 3. 


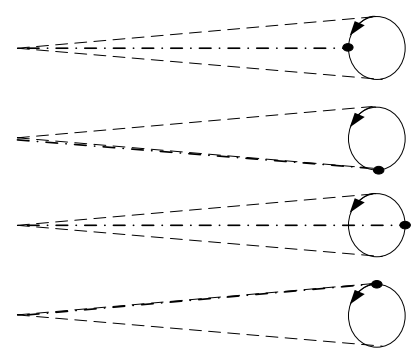

Fig.3 Coning motion of micro-machined gyroscope

The ground simulation experiments use the three-axis turntable to simulate the coning motion. The micro-gyroscope is mounted on the plate of the inner loop of the turntable. The inner loop rolls at the specific speed, the middle loop oscillates in the pitch direction, and the outer loop oscillates in the yaw direction.

In this experiment, the roll frequency of the inner loop is set at $15 \mathrm{~Hz}$. The middle and outer loops are set at $1 \mathrm{~Hz}, 5^{\circ}$. The results show in Fig. 4 and the insert (a), (b), (c), (d) and (F) illustrate the signal of transverse angular rate, the signal of azimuth angle, the signal of yaw angular rate, the signal of pitch angular rate and the signal of roll frequency which are demodulated from the signal of gyroscope.

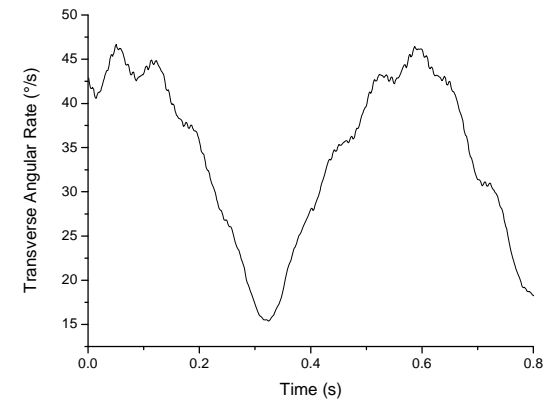

(a) Signal of transverse angular rate

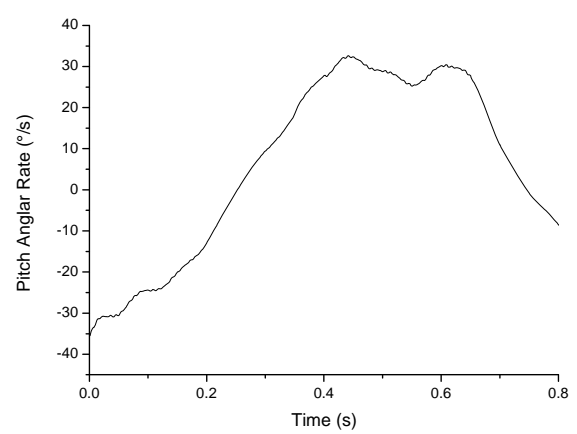

(c)Signal of yaw angular rate

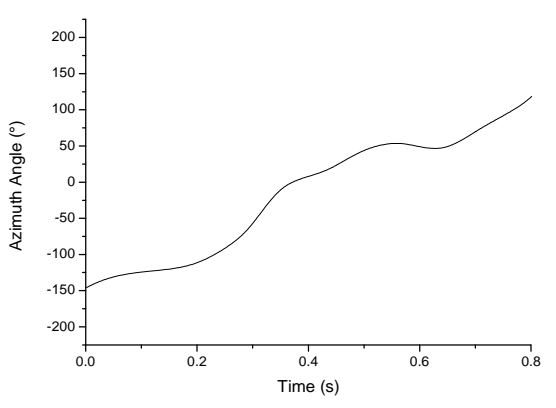

(b) Signal of azimuth angle

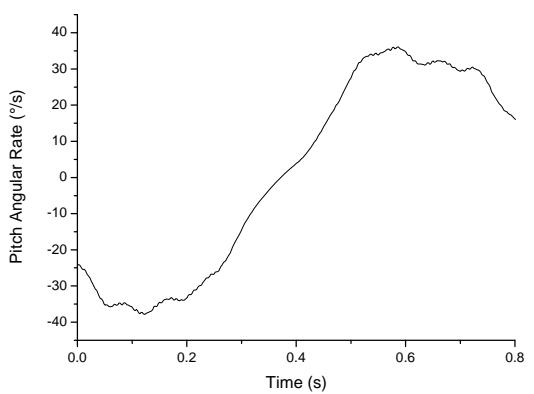

(d) Signal of pitch angular rate

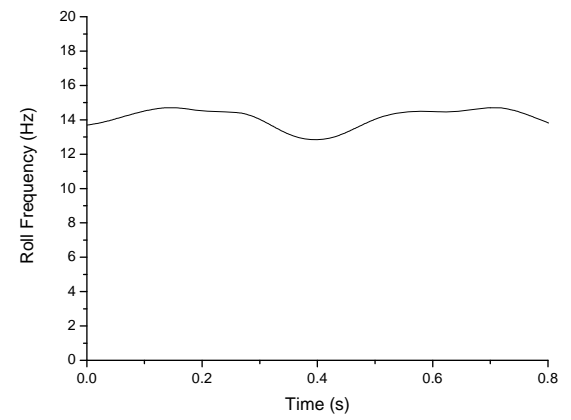

(e) Signal of roll frequency

Fig.4 Signal demodulation result of micro-machined gyroscope when the roll frequency of inner loop is $15 \mathrm{~Hz}$ 


\section{Conclusion}

This new gyroscope was designed to meet the requirement of rotating aircraft. The gyroscope can sense the roll of rotating aircraft and the transverse angular rate in 360 degree space. The gyroscope signal can be demodulated into the transverse angular rate, azimuth angle, yaw angular rate, pitch angular rate and roll frequency. The yaw and pitch angle error of demodulation is $6.43 \%$.

\section{Acknowledgment}

The work was funded by generous gift from National Natural Science Foundation of China Grant NSFC-60971024, Beijing Natural Science Foundation 4112020, Beijing Key Laboratory for Sensors 5026035204, and Key Laboratory of Modern Measurement \& Control Technology (Beijing Information Science \& Technology University), Ministry of Education.

\section{References}

[1] R. Hulsing, "MEMS inertial rate and acceleration sensor," IEEE AES Systems Magazine. 1998, 13(11): 17-23.

[2] A. Kourepenis, J. Bernstein, J. Connely, et al., "Performance of MEMS inertial sensors," Proc. IEEE Position Location and Navigation Symposium, 1998: 1-8.

[3] Y. Mochida, M. Tamura, and K. Ohwada, "A micromachined vibrating rate gyroscope with independent beams for the drive and detection modes" Proc., Twelfth IEEE Int. Conf. on Micro Electro Mechanical Systems (MEMS '99), Orlando, FL, 1999: 618-623.

[4] K. Funk, H. Emmerich, A. Schilp, et al., "A surface micromachined silicon gyroscope using a thick polysilicon layer," Proc., Twelfth IEEE Int. Conf. on Micro Electro Mechanical Systems (MEMS '99), Orlando, FL, 1999: 57-60.

[5] F. Zhang, Q. Yan, and W. Zhang, "Experiments and results of the silicon micromachined gyroscope," Journal of Micro-Nanolithography Mems and Moems, 2009, 8(2): 021130.

[6] Q. Yan, F. Zhang, L. Wu, "Theoretical and experimental study of silicon micro-machined gyroscope for measuring attitude of spinning projectile," .Piezoelectrics \& Acoustooptics, 2010, 32(2): 210-214,218.

[7] Q. Yan, W. Zhang, L. Wu, et al., "Measurement of rotation and angular vibration frequencies of spinning projectile in coning motion by a silicon micromachined gyro," Int. IEEE Conf. Inf. Autom., (ICIA09) June,2009, pp: 1018-1022. 